\title{
P03-11-1 Poster session
}

\section{The Impact of Health Technology Assessment on Pharmaceutical Decision Making}

\author{
Alima Almadiyeva ${ }^{1}$, Talgat Nurgozhin ${ }^{2}$, Alexander Kostyuk ${ }^{1}$ \\ ${ }^{1}$ Kazakhstan Association of Health Technologies Assessment, Evidence Based Medicine and Pharmacoeconomics \\ Research «KazSPOR», Kazakhstan, ${ }^{2}$ Asfendiyarov Kazakh National Medical University, Kazakhstan
}

Objectives: The aim of this study was to investigate pharmaceutical companies practices in relation to health technology assessment (HTA) and the views and perceptions of their executives on the importance of HTA in pricing and reimbursement of medicines in Kazakhstan. Methods: A qualitative study was performed, using individual semi-structured interviews based on an interview schedule with open-ended questions. The target population was market access departments' executives of originator pharmaceutical companies. Our target sample consisted of twenty executives, of whom ten agreed to participate. Saturation point was reached after twelve interviews. The survey included both closeended questions and an open-ended narrative section. Most of the closeended questions focused specifically on HTA, which was defined as 'a cluster of assessment and measurement techniques that aim to assess the relative value of a new medicine and commonly involve some form of economic measurement or measures of social well being. Space was provided on the questionnaire for respondents to write in comments for each close-ended question. Results: The discussion has been limited to survey questions specifically about HTA, including its influence on decisions about compounds in development and its impact on development costs and times. The survey also sought to define the impact of a broader range of factors on research and development. Participants considered HTA as a very important complementary tool for decision making in health policy, particularly in the field of pharmaceuticals and medical devices. They believed that, in Kazakhstan, HTA could be institutionalized for the reimbursement mechanism of medicines under certain conditions relating to current health policy-making attitudes and conditions pertaining in the country. They considered that there are many constraints which must be overcome as well as opportunities to be exploited.

Conclusions: Decisions in pharmaceutical policy should be scientifically substantiated and HTA should be institutionalized primarily for reimbursement decisions. Development of guidelines for conducting pharmacoeconomic evaluation, change in health policy goals, recording of cost and epidemiological data, and broader participation of all stakeholders in HTA decision-making processes are suggested as prerequisites for a successful implementation of HTA in Kazakhstan. 